\title{
EXPLORATION OF THE PARAMETER SPACE IN AN AGENT-BASED MODEL OF TUBERCULOSIS SPREAD: EMERGENCE OF DRUG RESISTANCE IN DEVELOPING VERSUS DEVELOPED COUNTRIES
}

\author{
AQUINO L. ESPINDOLA ${ }^{1, a}$, DANIEL GIRARDI ${ }^{1, b}$ \\ ${ }^{1}$ Departmento de Física, Instituto de Ciências Exatas-ICEx, Universidade Federal Fluminense \\ Volta Redonda, RJ, 27.213-250, Brazil \\ a aquinoespindola@id.uff.br, ${ }^{b}$ girardi@if.uff.br \\ THADEU J. P. PENNA ${ }^{1,2, c}$ \\ ${ }^{2}$ National Institute of Science and Technology for Complex Systems, Brazil \\ ${ }^{c}$ tjpp@if.uff.br \\ CHRIS T. BAUCH ${ }^{3, d}$ \\ ${ }^{3}$ Department of Mathematics and Statistics, University of Guelph, \\ N1G 2W1, Guelph, Ontario, Canada \\ dcbauch@uoguelph.ca \\ ALEXANDRE S. MARTINEZ ${ }^{2,4, e}$, BRENNO C. T. CABELLA ${ }^{4}$ \\ ${ }^{4}$ Faculdade de Filosofia, Ciências e Letras de Ribeirão Preto, Universidade de São Paulo, \\ Avenida dos Bandeirantes, 3900, 14.040-901, Ribeirão Preto, São Paulo, Brazil, \\ easmartinez@ffclrp.usp.br, ${ }^{f}$ brenno@usp.br \\ Received Day Month Year \\ Revised Day Month Year
}

\begin{abstract}
In this work we present an agent-based model for the spread of tuberculosis where the individuals can be infected with either drug-susceptible or drug-resistant strains and can also receive a treatment. The dynamics of the model and the role of each one of the parameters are explained. The whole set of parameters is explored to check their importance in the numerical simulation results. The model captures the beneficial impact of the adequate treatment on the prevalence of tuberculosis. Neverheless, depending on the treatment parameters range, it also captures the emergence of drug resistance. Drug resistance emergence is particularly likely to occur for parameter values corresponding to less efficacious treatment, as usually found in developing countries.
\end{abstract}

Keywords: Computational Epidemiology, Tuberculosis, Cellular Automata, Stochastic Models.

PACS Nos.: 87.23.Cc, 05.10.-a, 05.10.Ln 


\section{Introduction}

One third of the world population in infected with Mycobacterium tuberculosis, which causes the ancient disease Tuberculosis (TB) ${ }^{1}$. Along with this disease comes the emergence of the bacteria drug resistance due to the antibiotics treatment ${ }^{2}$. Several mathematical models $3,4,5,6,7,8,9,10,11$ have been developed to shed some light on the understanding of the TB epidemics, as well as the impact of the antibiotic use. Recently some of us have proposed a model of TB spread that includes local and global interactions using agents in a Monte Carlo simulation ${ }^{12}$. This model reproduces the results of the mean field equations from Refs $6,7,8,9$ for the limiting case where only global interactions are considered.

In this work, we aim to investigate whether drug resistance is more likely in developing or developed country settings therefore an extensive systematic study of the effect of the parameters of the previous model is relevant. It will also help us to understand sensitivity of model predictions to assumed parameter values.

This paper is organized as follows. In Sec. 2, we briefly describe our numerical simulation algorithm. In Sec. 3, we present our results. Finally in Sec. 4, we expose our conclusions and present some perspectives of future studies.

\section{The Model}

The tuberculosis spread numerical study is based on a stochastic agent-based model $^{12}$. In this model, the individuals are located in the sites of a square lattice and they are in one of the states of tuberculosis. The infection may be caused by sensitive, $S$, or resistant, $R$, pathogens. $S$ type bacteria is sensitive to the treatment with antibiotics and they may be completely cleared from the system by these drugs. On the other hand resistant type bacteria, $R$, may only be partially cleared by the antibiotics treatment.

Susceptible individuals, $X$, are those clear of pathogens. They may be infected by individuals $T_{i}$, which are infectious individuals with bacteria type $i$ and transmit the disease (infective state) since they are not under treatment. The index $i$ refer either $i=R$ or $i=S$ pathogen.

An individual in the $X$ state may be locally infected with probability ${ }^{13} P_{\mathcal{L}_{i}}=$ $1-\left(1-\beta_{i}\right)^{N_{T_{i}}}$, where $\beta_{i}$ is infectivity of type $i$ pathogen and $N_{T_{i}}$ is the amount of neighbours in the infectious state $T_{i}$. The infectivities are related by $\beta_{R}=\alpha \beta_{S}$, with $0<\alpha<1$ (see Refs. 14,15). This probability is evaluated for $i=S$ and $i=R$ neighborhood separately. The probability to occur a local infection due pathogens of type $S$ and $R$ is $P_{\mathcal{L}}=P_{\mathcal{L}_{S}}+P_{\mathcal{L}_{R}}-P_{\mathcal{L}_{S}} P_{\mathcal{L}_{R}}$. With this probability we are assuming co-infection and when a individual is infected with both bacteria types $(S$ and $R$ ) we assume that this individual is infected with bacteria type $R$. The infections of global origin due to both type of pathogens are assessed as follows: $P_{\mathcal{G}_{i}}=\beta_{i} T_{T_{i}} / N$. Again, this probability is evaluated for $S$ and $R$ neighborhood separately. The global probability is: $P_{\mathcal{G}}=P_{\mathcal{G}_{S}}+P_{\mathcal{G}_{R}}-P_{\mathcal{G}_{S}} P_{\mathcal{G}_{R}}$. Thus, the probability of that a susceptible individual, $X$, be infected by TB is $P=\Lambda P_{\mathcal{L}}+(1-\Lambda) P_{\mathcal{G}}$, where the parameter 
$\Lambda \in[0.0,1.0]$ adjusts the combination of local and global effects. If $\Lambda=1(0)$, only local (global) effects are taken into account in the infection process.

Latent individuals, $L_{S}$ and $L_{R}$, are those that host the TB pathogen, but they are not symptomatics. These individuals do not act as transmission agents of TB. If an individual is diagnosed as a carrier (latent) of the disease pathogen, he/she may be subjected to a prophylactic therapy called chemoprophylaxis. In our model a fraction $n_{L}$ of latent individuals, $L_{S}$ or $L_{R}$, may be submitted to a chemoprophylaxis therapy. After the therapy time, individuals may be cured with probability $\sigma$, progress to either $T_{S}$ or $T_{R}$ states with probability $v$, or remain latent. Those who do not receive chemoprophylaxis therapy may progress to active states $T_{S}$ or $T_{R}$ with probability $v$.

Parameters that govern the dynamics of the model are: $\beta_{R}$, the infectivity of type $R$ pathogens; $n_{L}$, the fraction of latent individuals that receive chemoprophylaxis; $n_{T}$, the fraction of infectious individuals that receive treatment; $\delta$, relative treatment efficacy for type $R$ bacteria; $\Lambda$, relative importance of local/global effects. There are also the probabilities of natural death, $\mu$; death due to tuberculosis, $\mu_{T}$; developing active tuberculosis from $X$ state, $p$; disease progression (latent to infectious) in latent individuals, $v$; chemoprophylaxis therapy is effective, $\sigma$; effective treatment for infectious individuals, $\phi$; developing drug resistance during treatment, $r$.

A proportion $n_{T}$ of infectious individuals receive treatment with antibiotics. For $T_{S}$ individuals under treatment, three outcomes are possible: the treatment may be successful with probability $(1-r) \phi$; treatment fails and emergence of drug resistance may happen with probability $r \phi$; or finish the period of treatment with no cure but also without emergence of drug resistance with probability $1-\phi$. This dynamics also applies to infectious individuals that host the type $R$ bacteria, $T_{R}$. The only difference is that efficacy of antibiotics treatment is reduced by a factor $\delta$. Thus, the efficacy of antibiotics for $T_{R}$ cases is $\delta \phi$.

For the sake of clarity, parameters of the model ${ }^{12}$ are summarized in Table 1.

\section{Results}

Here, we present the results of the parameter space exploration. For all runs the averages were obtained over 30 realizations of the system. We have simulated an 100,000 inhabitants population ${ }^{12}$, as usual in this kind of work. Unless explicitly mentioned, in all numerical experiments, the parameters are: $L=317, \mu_{T}=2.74 \times$ $10^{-4} /$ day, $\mu=3.65 \times 10^{-5} /$ day, $\delta=0.8, p=1.37 \times 10^{-4} /$ day, $v=3.13 \times 10^{-5} /$ day, $r=0.5, \beta_{S}=2.47 \times 10^{-3} /$ day, $n_{T}=0.6, n_{L}=0.1$ and $\alpha=0.8$.

The treatment efficacy, $\phi$, the chemoprophylaxis efficacy, $\sigma$, and probability to develop drug resistance during treatment $r$ are parameters that depend mostly on the health policies of each country. As a rule of thumb, the more developed the country is, the more is invested in health and for this reason developed (developing) present better (worse) results for $\phi, \sigma$ and $r$. Thus, this set of parameters, $(\phi, \sigma, r)$, will be considered as a measure of the country development level. 


\begin{tabular}{c|l}
\hline Parameter & Definition \\
\hline$\beta_{i}$ & infectivity of type $i$ pathogen, with $i=S, R$ \\
$\alpha$ & relative infectivity between type $S$ and $R$ pathogen $(0<\alpha<1)$ \\
$\Lambda$ & adjustment of local and global effects $(\Lambda \in[0.0,1.0])$ \\
$n_{L}$ & proportion of latent individuals that receive chemoprophylaxis \\
$n_{T}$ & proportion of infective individuals that receive treatment \\
$\delta$ & relative treatment efficacy \\
$\mu$ & probability of natural death \\
$\mu_{T}$ & probability of death due to tuberculosis \\
$p$ & probability of developing active tuberculosis from $X$ state \\
$v$ & probability of disease progression in latent individuals \\
$\sigma$ & probability that chemoprophylaxis therapy is effective \\
$\phi$ & probability of effective treatment for infectious individuals \\
$r$ & probability to develop drug resistance during treatment \\
\hline
\end{tabular}

Table 1: Parameters of the model.

In figures 1a-1d, one can see the annual disease incidence of latent cases, $L_{S}$ and $L_{R}$, as a function of the proportion of latent individuals that receive chemoprophylaxis, $n_{L}$. Figures $1 \mathrm{a}$ and $1 \mathrm{~b}$ present the incidence of latent cases with type $S$ bacteria as a function of $n_{L}$ for developed and developing countries, respectively. Both plots present a similar behavior, with exception only for $n_{L} \approx 0$. Once the chemoprophylaxis efficacy does not depend on the $\phi$ value, it is expected that results are the same for developed and developing countries. For values of $n_{L}>0.1$, the amount of latent individuals vanishes independently of the $\sigma$ value. Thus, for cases of latency with sensitive pathogens, the chemprophylactic treatment has a high impact on the bacterial clearance. Cases of latency with type $R$ pathogens as a function of $n_{L}$ can be seen in the plots of Figs. $1 \mathrm{c}$ and $1 \mathrm{~d}$. Recall that in our model, chemoprophylaxis does not clear type $R$ bacteria, independently of the efficacy $\sigma$. Therefore, as $n_{L}$ increases, only $L_{S}$ individuals are cured and $L_{R}$ individuals remain in the population. Thus, in the contagion process competition between $S$ and $R$ strains disappears. For this reason in both developed and developing countries the elimination of $S$ pathogens due to chemoprophylaxis helps $R$ strains to spread.

Figures $2 \mathrm{a}-2 \mathrm{~d}$ depict infective cases of tuberculosis, $T_{S}$ and $T_{R}$, as a function of $n_{L}$ for different $\sigma$ values. Again, the characterization of a country as developed or developing is determined by the treatment efficacy, $\phi$. For $T_{S}$ cases, the plots of Figs. $2 \mathrm{a}$ and $2 \mathrm{~b}$, independently of the $\sigma$ values, the total disease clearance occurs for $n_{L} \approx$ 0.1 , for developed and developing countries. This phenomenon is explained by the fact that chemoprophylactic therapy rapidly cures latent individuals $L_{S}$. Therefore, the progression of the disease from latent state to infectious state is interrupted. Considering a fixed value of $n_{L}$, the increasing of $\sigma$ has an effect of reduction on the annual disease incidence. In its turn, $T_{R}$ cases plotted in Figs. $2 \mathrm{c}$ and $2 \mathrm{~d}$, tend to increase as $n_{L}$ is raised. As mentioned above, chemoprophylactic therapy clears only 


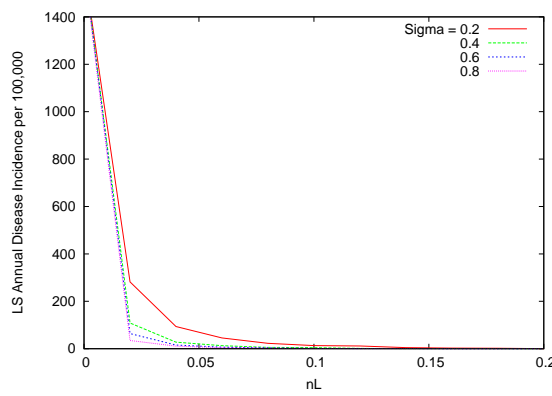

(a) $L_{S}:$ developed countries.

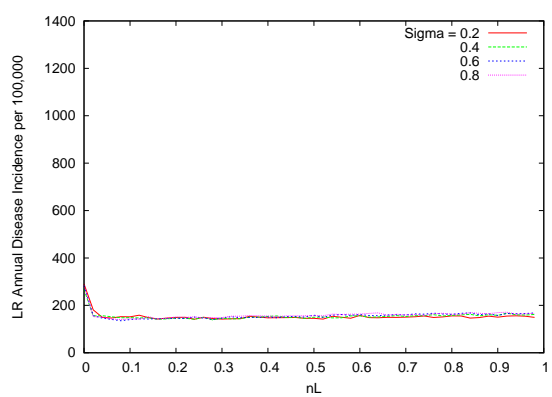

(c) $L_{R}$ : developed countries.

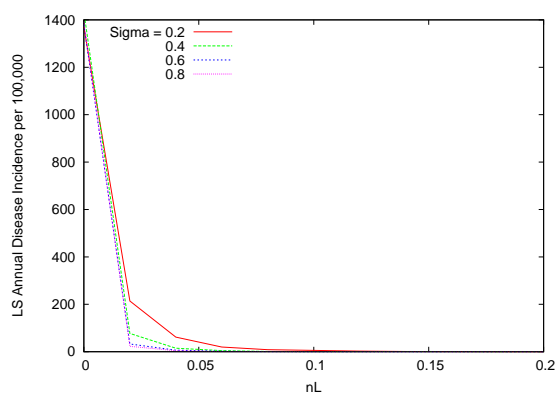

(b) $L_{S}$ : developing countries.

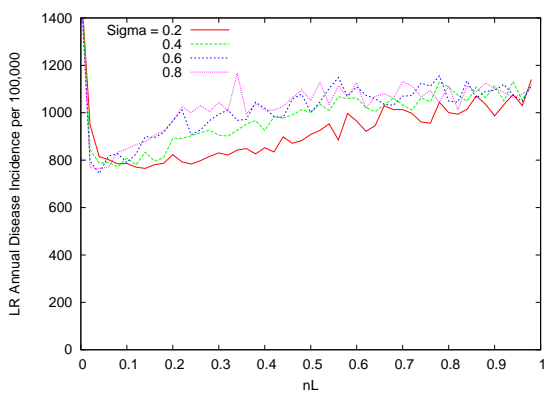

(d) $L_{R}$ : developing countries.

Fig. 1: $L_{S}$ and $L_{R}$ cases as a function of $n_{L}$ for different values of $\sigma=0.2,0.4,0.6,0.8$. Parameters are $(\phi=0.95, r=0.1)$ for developed countries, and $(\phi=0.5, r=0.7)$ for developing countries. Error bars have the same magnitude of the mean values.

latent cases with type $S$ pathogen. For this reason, as $n_{L}$ increases, the pool of latent individuals $L_{R}$ also increases. Consequently, the rate of progression to the infectious state $T_{R}$ is also increased. The annual incidence of $T_{R}$ cases presents higher values for developing countries, as shown in Fig. 2d. The lower treatment efficacy, $\phi=0.5$, favors the emergence of drug resistance and consequently the spread of resistant strains.

Figures $3 \mathrm{a}$ and $3 \mathrm{~d}$ show $T_{S}$ and $T_{R}$ cases as a function of the proportion of infectious individuals that receive treatment, $n_{T}$. In both plots, different curves represent three different treatment efficacies: $\phi=0.5,0.7,0.9$. For the sake of clarity, Figs. 3a-3d have insets with a zoom for $n_{T}=0.5$ to $n_{T}=1.0$.

Figures $3 \mathrm{a}$ and $3 \mathrm{~b}$ one sees that as $n_{T}$ increases, the amount of $T_{S}$ individuals decreases. For the three treatment efficacy values, all $T_{S}$ cases almost vanish from the population for $n_{T}$ around 0.3. The higher $\phi$, the faster the decay of $T_{S}$ individuals in the population, as expected. The drug resistant cases, $T_{R}$, as a function of $n_{T}$, can be seen in the plots of Figures $3 \mathrm{c}$ and $3 \mathrm{~d}$. Note that for the three curves, the drug resistance emergence has a peak in $n_{T}$ around 0.05 . The combination of two concurrent effects explains the peaks in the plots of Figs. 3c and 3d: the pool 


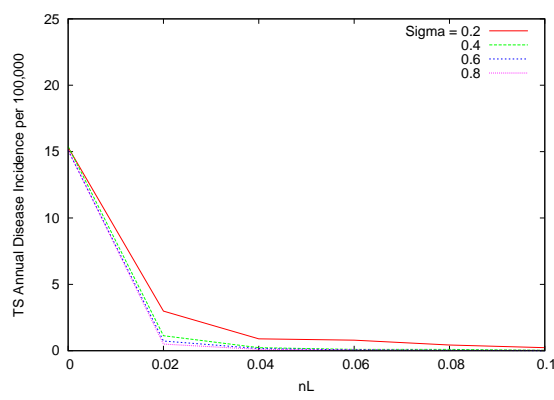

(a) $T_{S}$ : developed countries.

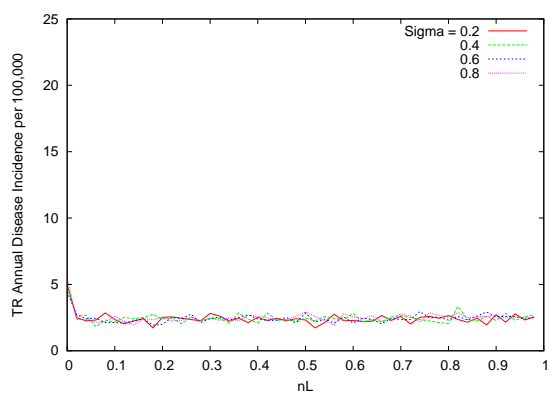

(c) $T_{R}$ : developed countries.

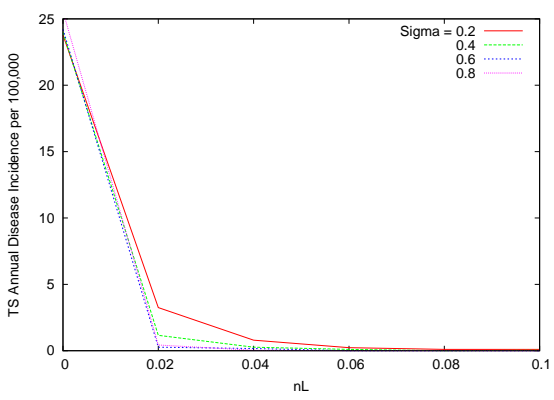

(b) $T_{S}$ : developing countries.

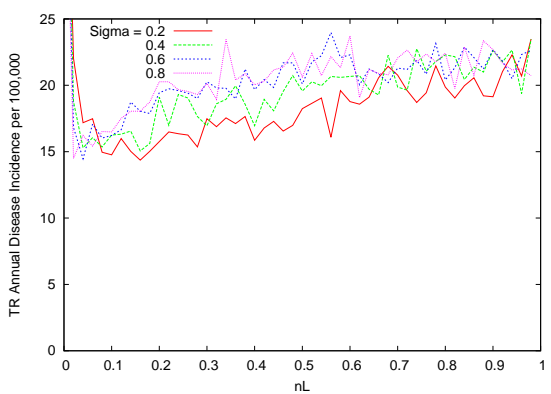

(d) $T_{R}$ : developing countries.

Fig. 2: $T_{S}$ and $T_{R}$ cases as a function of $n_{L}$ for different values of $\sigma=0.2,0.4,0.6,0.8$. Parameters are $(\phi=0.95, r=0.1)$ for developed countries, and $(\phi=0.5, r=0.7)$ for developing countries. Error bars have the same magnitude of the mean values.

of $T_{S}$ individuals in the population, and the probability of treatment failure, $r \phi$.

Increasing $n_{T}$ means that the amount of $T_{S}$ individuals under treatment is also increased. Thus, the number of treatment failures, i.e, emergence of drug resistance is also higher. Therefore, even though the pool of $T_{S}$ cases is reduced, the pool of $T_{R}$ individuals is incremented. This behavior occurs in the region of $0<n_{T} \lesssim 0.05$. For $n_{T} \gtrsim 0.05, T_{R}$ cases rapidly vanish. Again, the higher $n_{T}$ is, the higher the amount of $T_{S}$ individuals under treatment that are cured. In this case, the pool of $T_{S}$ (figs. $3 \mathrm{a}$ and $3 \mathrm{~b}$ ) is reduced to values that do not favor an increase in the emergence of drug resistance.

As described in the Sec. 2, the probability that a susceptible individual, $X$, is infected by $\mathrm{TB}$ is ${ }^{12}$ :

$$
P=\Lambda P_{\mathcal{L}}+(1-\Lambda) P_{\mathcal{G}},
$$

where $P_{\mathcal{L}}$ and $P_{\mathcal{G}}$ are the local and global probabilities of contagion, respectively. In Eq. (1), parameter $\Lambda \in[0.0,1.0]$ adjusts the combination of local and global effects.

The impact of local and global effects in the spread of TB for developed and developing countries are plotted in Fig. 4 . In this plot, $\Lambda$ varies from $\Lambda=0$, only 


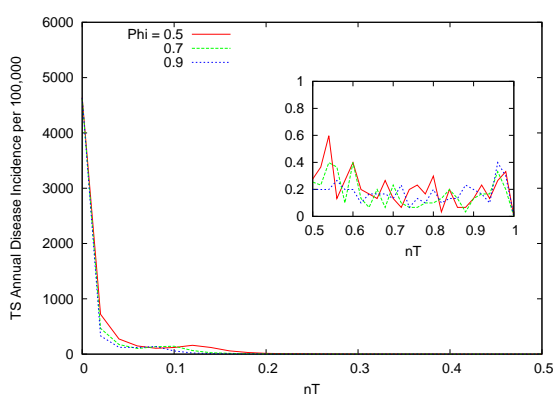

(a) $T_{S}$ : developed countries.

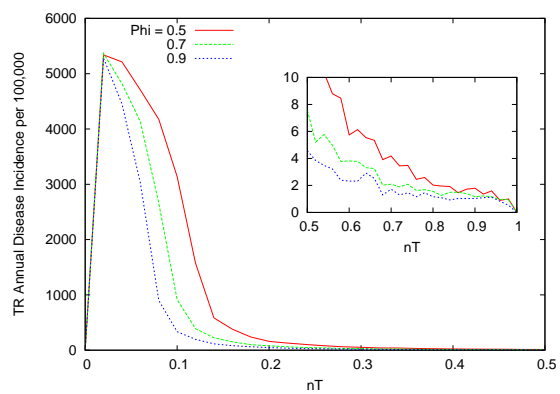

(c) $T_{R}$ : developed countries.

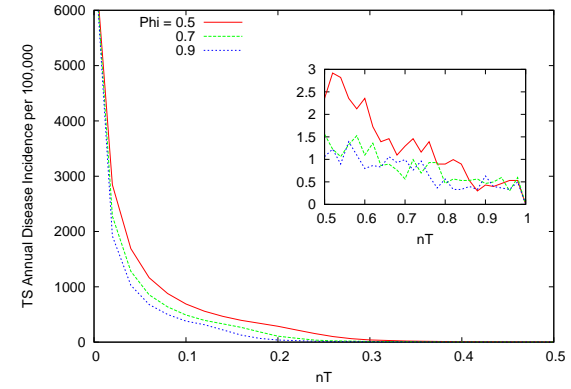

(b) $T_{S}$ : developing countries.

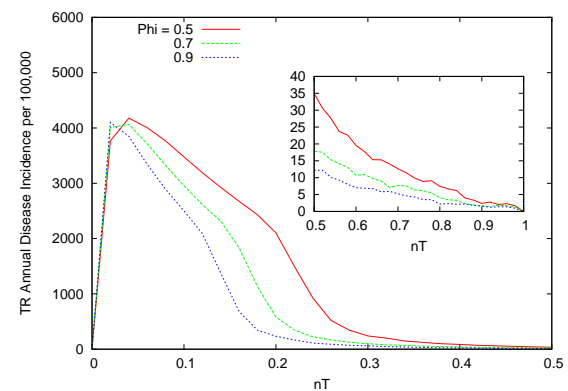

(d) $T_{R}$ : developing countries.

Fig. 3: $T_{S}$ and $T_{R}$ cases as a function of $n_{T}$ for different values of $\phi$. Parameters are $(\sigma=$ $0.2, r=0.1)$ for developed countries, and $(\sigma=0.05, r=0.7)$ for developing countries. Insets: in all figures are a zoom from $n_{T}=0.5$ to $n_{T}=1.0$. Error bars have the same magnitude of the mean values.

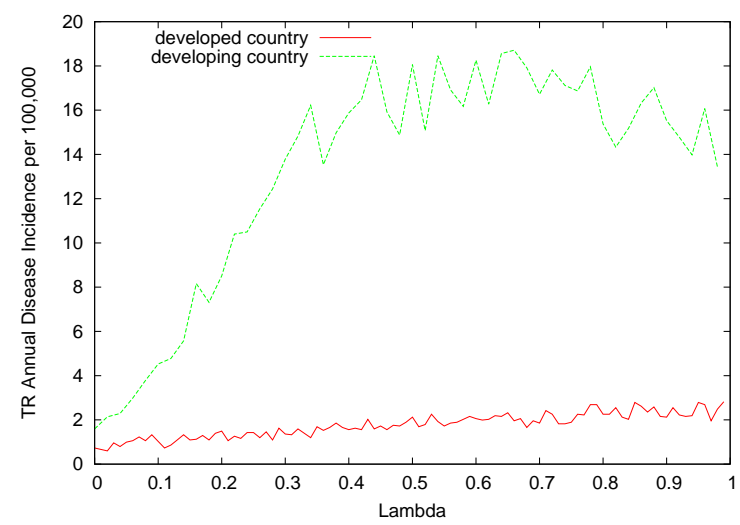

Fig. 4: $T_{R}$ cases as a function of $\Lambda$ for developed countries $(\phi=0.95, \sigma=0.2, r=0.1)$ and developing countries $(\phi=0.5, \sigma=0.5, r=0.5)$. Error bars have the same magnitude of the mean values. 
global effects, up to, $\Lambda=1$, only local effects. Both curves, developed and developing countries, present a similar behavior, i.e, the incidence of $T_{R}$ increases as $\Lambda$ increases. This behavior clearly indicates that local effects are more important in the contagion process.

\section{Conclusion}

We have presented the exploration of the parameter space of an agent-based model of tuberculosis spread, which is briefly reviewed. One of the most interesting result is the dependence of the number of infected individuals on the health policies. Even for a small fraction of the population in the latent state under treatment, the results suggest a remarkable decrease of the number of individuals infected with the most aggressive strain for both levels of development. However, since chemoprophylaxis has different efficacies for the two strains, by not clearing one of them, we have found a counter intuitive result of increasing number of latent individuals with type $R$, with the number of individuals under treatment. This effect is more pronounced in countries under development than those with a higher level of development.

\section{Acknowledgments}

Authors thank anonymous referees for the comments and their constructive suggestions. A.L.E. and C.T.B. wish to thank FAPESP, Natural Sciences and Engineering Research Council of Canada (NSERC) and Canadian Institutes of Health Research (CIHR) for financial support. D. Girardi thanks CNPq and National Institute of Science and Technology for Complex System - Brazil. ASM acknowledges support from CNPq (305738/2010-0 and 476722/2010-1).

\section{References}

1. Tuberculosis Fact sheet Nr. 104. World Health Organization. November 2010. http://www.who.int/mediacentre/factsheets/fs104/en/index.html. Retrieved 26 July 2011.

2. D. J. Austin et al. The relationship between the volume of antimicrobial consumption in human communities and the frequency of resistance. PNAS, 96:1152-1156, 2007.

3. Z. Feng L.-I. Roeger and C. Castillo-Chavez. Modelling TB and HIV coinfections. Mathematical Biosciences and Engineering, 6:817-839, 2009.

4. C. Castillo-Chavez and Z. Feng. To treat or not treat the case of tuberculosis. J. Math. Biol., 35(6):629-656, 1997.

5. Castillo-Chaves Z. Feng, C and A. F. Capurro. A model for tuberculosis with exogenous reinfection. Theor. Popul. Biol., 57(3):235-247, 2000.

6. S. M. Blower and J. L. Gerberding. Understanding, predicting and controlling the emergence of drug-resistant tuberculosis: a theoretical framework. J. Mol. Med., 76:624-636, 1998.

7. S. M. Blower et al. The intrinsic transmission dynamics of tuberculosis epidemics. Nature Medicine, 8(1):815-821, 1995.

8. P. M. Small S. M. Blower and P. Hopewell. Control strategies for tuberculosis epidemics: new models for old problems. Science, 273:497-500, 1996. 
9. T. C. Porco T. S. M. Blower and T. Lietman. Tuberculosis: the evolution of antibiotic resistance and the design of epidemic control strategies. Mathematical Models in Medical and Health Sciences. Eds Horn, Simonett, Webb. Vanderbilt University Press, 1998.

10. Christopher J. L. Murray and Joshua A. Salomon. Modeling the impact of global tuberculosis control strategies. PNAS, 95(23):13881-13886, 1998.

11. E. E. Salpeter and S. R. Salpeter. Mathematical model for the epidemiology of tuberculosis, with estimates of the reproductive number and infectious-delay function. Am. J. Epidemiol., 147(4):398-406, 1998.

12. Aquino L de Espindola, Chris T. Bauch, Brenno C. T. Cabella and Alexandre S. Martinez. An agent-based computational model of the spread of tuberculosis. Journal of Statistical Mechanics: Theory and Experiment, page P05003, 2011.

13. V. J. Haas D. Alves and A. Caliri. The predictive power of $R_{0}$ in an epidemic probabilistic system. Journal of Biological Physics, 29:63-75, 2003.

14. L. H. Schimdt at al. The emergence of isoniazid-sensitive bacilli in monkeys inoculated with isoniazid-resistant strains. Trans. 17th Conference on Chemotherapy of Tuberculosis - VA Armed Forces, page 264, 1958.

15. B. Sommers T. Cohen and M. Murray. The effect of drug resistance on the fitness of mycobacterium tuberculosis. Lancet Infec. Dis., 3(1):13-21, 2003. 